\title{
APERIODIC TILINGS IN HIGHER DIMENSIONS
}

\author{
CHARLES RADIN
}

(Communicated by Barbara L. Keyfitz)

\begin{abstract}
We show that in dimensions $d \geq 3$, aperiodic tilings can naturally avoid more symmetries than just translations.
\end{abstract}

\section{INTRODUCTION}

The subject known as aperiodic tilings developed from the following phenomenon. For a subshift of finite type $X \subseteq A^{\mathbb{Z}^{d}}$ :

Elementary Lemma. If $d=1, X$ must contain a configuration invariant under a nontrivial translation.

Theorem 1 [2]. If $d \geq 2, X$ need not contain a configuration invariant under a nontrivial translation.

The above phenomenon was discovered through a question by the philosopher Hao Wang in $1961[16,17]$ and the answer by his student Robert Berger [2] in 1966. The subject of aperiodic tilings has evolved in the ensuing decades as the study of the properties of systems of finite type not containing any configuration with a translation symmetry. There have been three lines of development, examining what, beyond translation symmetry, can be avoided by all configurations of a system of finite type. One line was concerned with complexity, and showed the existence of $X$ such that no configuration in $X$ is a computable function of $j \in \mathbb{Z}^{d}$ [7]. One line concerned disorder, and has shown the existence of weakly mixing $X[9,1]$. The existence of strongly mixing $X$ remains an important open question $[10,1]$; we note that mixing is closely associated with the spectrum of the dynamical system. The largest effort, concerned with symmetry, followed after Wang's problem was generalized (by Roger Penrose et al. $[5,6])$ from subshifts of finite type (corresponding to tiles which are basically cube-shaped) to "tiling dynamical systems", with tiles of rather general shape (though there is no loss of generality in assuming they are polyhedra). For these systems attention again focused on the spectrum of the dynamical system, this time on its symmetry with respect to the natural action of the rotations of $E^{d}$. For a review see $[10,13]$.

Received by the editors October 27, 1993 and, in revised form, April 8, 1994.

1991 Mathematics Subject Classification. Primary 58F11, 52C20, 47A35.

Research supported in part by NSF Grant No. DMS-9304269 and Texas ARP Grant 003658113. 
In this paper we consider an old part of the subject, its association with substitution dynamics, and in particular the way in which the subject treats tilings in various dimensions. One consequence of our main result is to question the traditional aims of "aperiodicity" as discussed above; we conclude that the traditional concentration on avoiding translations has been overly restrictive and due in part to the fact that in 2 dimensions the only (orientation-preserving) congruences without fixed points are the translations. We will see that by emphasizing the role of substitutions the other congruences which can appear in higher dimensions can play a natural role in the subject.

A tiling dynamical system of dimension $d, X_{F}$, which will carry the action of the group of translations on $E^{d}$, is defined as follows. Let $A$, the "alphabet", be a finite nonempty collection of "letters", that is, pairwise noncongruent polyhedra in $E^{d}$, and let $K$ be the maximum of the diameters of the letters. Consider the connected subgroup $G$ of the Euclidean group of (maps on) $E^{d}$, presented in the form of a semidirect product $G=T \otimes_{s} R$ of the subgroups $T$ of translations and $R$ of rotations (about the point $0 \in T \cong E^{d}$ ); $(t, r)\left(t^{\prime}, r^{\prime}\right)=\left(t+r\left[t^{\prime}\right], r r^{\prime}\right)$. We denote by $e$ the identity in $R$. We define a "tile" as the image $(t, r) P$ of a letter $P$ by some $(t, r) \in G$, and we define a "patch" (or "word") as a finite collection $x=\left\{x_{i}\right\}$ of tiles for which: the union is a connected and simply connected set and the interiors $\operatorname{Int}\left(x_{i}\right)$ are pairwise disjoint. Using the notation $\bar{B}_{t}^{d}(m)$ for the closed ball of radius $m$ centered at $t \in E^{d}$, we assume given a finite nonempty set, $F$, of patches, each patch having the following properties:

(a) the origin is a vertex of one of the tiles of the patch;

(b) the union of the tiles contains $\bar{B}_{0}^{d}(1)$;

(c) $r(P) \cap \bar{B}_{0}^{d}(1)=Q \cap \bar{B}_{0}^{d}(1)$, where $P, Q \in F$ and $r \in R$, implies $P=Q$.

Finally, we define $X_{F}$ as the set of all countable sets ("tilings") $x=\left\{x_{i}\right\}$ of tiles such that:

(i) $\bigcup_{i} x_{i}=E^{d}$ (the "covering condition");

(ii) $\operatorname{Int}\left(x_{i}\right) \cap \operatorname{Int}\left(x_{j}\right)=\varnothing$ for $i \neq j$ (the "packing condition");

(iii) if $t$ is a vertex in $x$, then there is some $r \in R$ such that $[(0, r)(-t, e) x]$ $\cap \bar{B}_{0}^{d}(1) \in F$ (the "finite type condition").

(There is a natural topology for $X_{F}$, similar to the product topology of symbolic dynamical systems, such that $X_{F}$ is compact and metrizable, and such that the action of $G$ on $X_{F}$ is jointly continuous; we will not need it for our results, so we just refer to $[14,12,13]$ for details.)

\section{Results}

We begin with an example due to Peter Schmitt [15], a 3-dimensional tiling dynamical system $X_{F}$. The key feature is that $X_{F}$ is generated by a tiling which, though not invariant under any translation, is invariant under a "screw motion" in $E^{3}$, the composition of a translation with a rotation (in fact irrational) about an axis parallel to the translation. (These tilings have been improved cosmetically by J. Conway and by L. Danzer - see [4].)

Intuitively this tiling seems less interesting than the earlier ones of Berger, Penrose, et al. We will attempt to clarify the distinction with the help of the 
following subshift. Let $S$ be the 1-torus, $\left\{e^{i \alpha}: \alpha \in[0,2 \pi)\right\}$, and

$$
X=\left\{x \in S^{\mathbb{Z}}: x_{j+1}=e^{i} x_{j} \text { for all } j \in \mathbb{Z}\right\} .
$$

$X$ is a much simpler "version" of Schmitt's example; it too is generated by one element, which is not invariant under any translation, but which is invariant under the symmetry $\tau: X \rightarrow X$ defined by $(\tau x)_{j}=e^{-i}\left(x_{j+1}\right)$. In other words, by allowing an infinite set $S$ in this one-dimensional subshift we can no longer conclude the existence of a configuration with a translation symmetry, although the slightly more complicated symmetry $\tau$ seems to play the same role.

Theorem 1, which shows that translation symmetry can be avoided by subshifts of finite type in dimensions at least 2, was proven by Berger using a hierarchical symmetry of expanding squares. Using Schmitt's 3-dimensional example as inspiration, and the above crude one-dimensional analogue of it, we see that avoiding translation symmetry is not the proper focus of this new phenomenon, and are thus led to consider the following result which uses hierarchical symmetry to avoid not just translations but other geometric symmetries as well.

Theorem 2. Suppose $X$ is a G-invariant subset of the d-dimensional tiling $d y$ namical system $X_{F}, d \geq 2$, and assume there is some $\tilde{x} \in X_{F}$ such that $(\tilde{t}, \tilde{r}) \tilde{x}=\tilde{x}$ for some $(\tilde{t}, \tilde{r}) \in G$. Also assume there is some $0<\delta<1$, and an invertible map $D$ on $X$ such that for all $x \in X, D[(t, r) x]=(\delta t, r) D[x]$ for all $(t, r) \in G$. Then $(\tilde{t}, \tilde{r})$ leaves fixed some point of $E^{d}$.

Proof. We will restrict attention to the case in which $\tilde{r} \neq e$, the proof for the rest being very easy to obtain from this case.

We begin with the spectral decomposition of normal maps on the real inner product space $E^{d}$. Given $r \in R, r \neq e, E^{d}$ is the direct sum of pairwise orthogonal $r$-invariant subspaces, $E^{d}=\bigoplus_{j=1}^{p} S_{j}, p \geq 2$, where on $S_{1}$ (possibly zero-dimensional if $d$ is even) the restriction $r_{1}$ of $r$ is the identity map $e_{1}$, the other $S_{j}$ are each 2-dimensional, and on each of these subspaces the restriction $r_{j}$ of $r$ is a nontrivial rotation. Given this, consider now the map $(\tilde{t}, \tilde{r})$ on $E^{d}$. Decomposing $\tilde{t}$ into its projections $\tilde{t}_{j}$ in the $S_{j}$, we have the decomposition $(\tilde{t}, \tilde{r})=\sum_{j=1}^{p}\left(\tilde{t}_{j}, \tilde{r}_{j}\right)$, where $\tilde{r}_{1}=e_{1}$, and, for $j \geq 2,\left(\tilde{t}_{j}, \tilde{r}_{j}\right)$ is, within the space $S_{j}$, just rotation about $\left(e_{j}-\tilde{r}_{j}\right)^{-1}\left(\tilde{t}_{j}\right)$. In summary, for $s=\sum_{j=1}^{p} s_{j} \in E^{d}$,

$$
(\tilde{t}, \tilde{r}) s=\left(\tilde{t}_{1}+s_{1}\right)+\sum_{j=2}^{p}\left\{\tilde{r}_{j}\left[s_{j}-\left(e_{j}-\tilde{r}_{j}\right)^{-1} \tilde{t}_{j}\right]+\left(e_{j}-\tilde{r}_{j}\right)^{-1} \tilde{t}_{j}\right\}
$$

and so for $m \in \mathbb{N}$

$$
(\tilde{t}, \tilde{r})^{m} s=\left(k \tilde{t}_{1}+s_{1}\right)+\sum_{j=2}^{p}\left\{\tilde{r}_{j}^{m}\left[s_{j}-\left(e_{j}-\tilde{r}_{j}\right)^{-1} \tilde{t}_{j}\right]+\left(e_{j}-\tilde{r}_{j}\right)^{-1} \tilde{t}_{j}\right\} .
$$

We need to show that $\tilde{t}_{1}=0$, yielding the fixed point $\sum_{j=2}^{p}\left(e_{j}-\tilde{r}_{j}\right)^{-1} \tilde{t}_{j}$.

Let $x_{1} \equiv D[\tilde{x}]$. Then

$$
D\left[(\tilde{t}, \tilde{r}) D^{-1}\left[x_{1}\right]\right]=D[(\tilde{t}, \tilde{r}) \tilde{x}]=D[\tilde{x}]=x_{1} .
$$

But $D(\tilde{t}, \tilde{r}) D^{-1}=(\delta \tilde{t}, \tilde{r})$, so $(\delta \tilde{t}, \tilde{r}) x_{1}=x_{1}$. Iterating the argument, if $x_{n} \equiv D^{n}[\tilde{x}]$ we have $\left(\delta^{n} \tilde{t}, \tilde{r}\right) x_{n}=x_{n}$. 


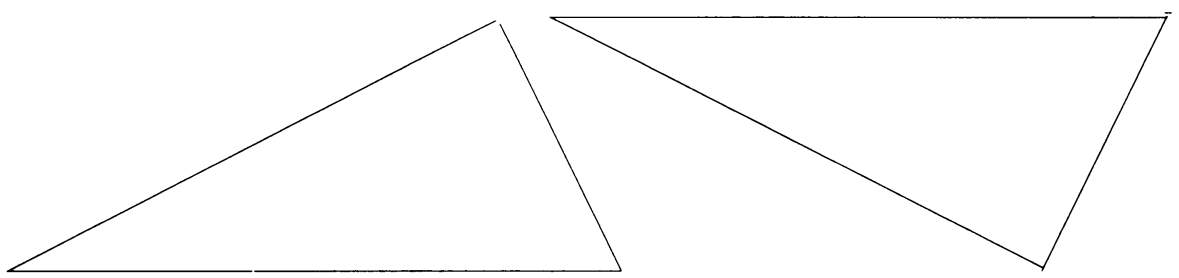

FigURE 1

Let $w$ be half the minimum separation between verices in the letters. For any dimension $d$ and point $t \in E^{d}$ it follows by compactness that for every point $t^{\prime \prime}$ in $\bar{B}_{t}^{d}(K)$ there are positive integers $m_{1}<m_{2}$ such that

$$
\left\|\left(\tilde{r}^{m_{2}}\left[t^{\prime \prime}-t\right]+t\right)-\left(\tilde{r}^{m_{1}}\left[t^{\prime \prime}-t\right]+t\right)\right\|<w / 2 .
$$

We apply this to $t=\sum_{j=2}^{p}\left(e_{j}-\tilde{r}_{j}\right)^{-1} \delta^{n} \tilde{t}_{j}$, where $n$ is such that

$$
\left\|\left(m_{2}-m_{1}\right) \delta^{n} \tilde{t}_{1}\right\|<w / 2 .
$$

If $t^{\prime \prime}$ is a vertex in $x_{n}$, with $t^{\prime \prime} \in \bar{B}_{t}^{d}(K)$ (and by the definition of $K$ there must be such a point in $x_{n}$ ), then since, as in (3), for $m \in \mathbb{N}$

(7) $\left(\delta^{n} \tilde{t}, \tilde{r}\right)^{m} t^{\prime \prime}=m \delta^{n} \tilde{t}_{1}+t_{1}^{\prime \prime}+\sum_{j=2}^{p}\left\{\tilde{r}_{j}^{m}\left[t_{j}^{\prime \prime}-\left(e_{j}-\tilde{r}_{j}\right)^{-1} \delta^{n} \tilde{t}_{j}\right]+\left(e_{j}-\tilde{r}_{j}\right)^{-1} \delta^{n} \tilde{t}_{j}\right\}$,

it follows using (5) and (6) that $\left\|\left(\delta^{n} \tilde{t}, \tilde{r}\right)^{m_{2}} t^{\prime \prime}-\left(\delta^{n} \tilde{t}, \tilde{r}\right)^{m_{1}} t^{\prime \prime}\right\|<w$. Therefore $\left(\delta^{n} \tilde{t}, \tilde{r}\right)^{m_{1}} t^{\prime \prime}=\left(\delta^{n} \tilde{t}, \tilde{r}\right)^{m_{2}} t^{\prime \prime}$, which implies that $\tilde{t}_{1}=0$.

In Theorem 2 the key assumption is the map $D$, which is a nontrivial representation on $X$ of a similarity of $E^{d}$; the canonical illustrative example is the pinwheel tilings $[11,12,13]$, defined as follows.

The alphabet $A$ consists of the two triangles in Figure 1. The "substitution rule" for $A$ consists of the dilation $\tilde{D}$ about the origin of $E^{2}$ by the factor $\delta=1 / \sqrt{5}$, and a set $\left\{C_{j k}: 1 \leq j \leq 2,1 \leq k \leq 5\right\}$ of elements of $G$, such that for each $P_{j} \in A$ we have:

$$
P_{j}=\bigcup_{k} C_{j k} \tilde{D} P_{n_{k}}
$$

where the elements of each union have pairwise disjoint interiors. (See Figure 2.) The "substitution function" $D$ associated with the rule is defined on tiles (and then sets of tiles), with sets of tiles as values, as follows. If the tile $P$ is "of tile-type $j$ ", that is, $P=C P_{j}$ where $P_{j} \in A$ and $C \in G$, then

$$
D: P \longrightarrow D(P) \equiv\left\{\tilde{D}^{-1} C C_{j k} \tilde{D} P_{n_{k}}\right\} .
$$

(Intuitively, $P$ is replaced by a set of "small-size tiles" by (8), which are then expanded in (9) to original size by the inverse of the dilation. This process can obviously be applied to any collection of tiles, for example a tiling, and can thus be iterated.) 


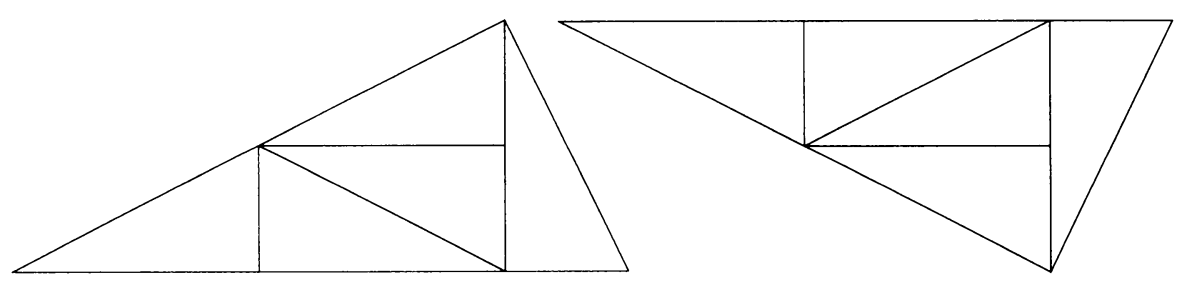

FIGURE 2

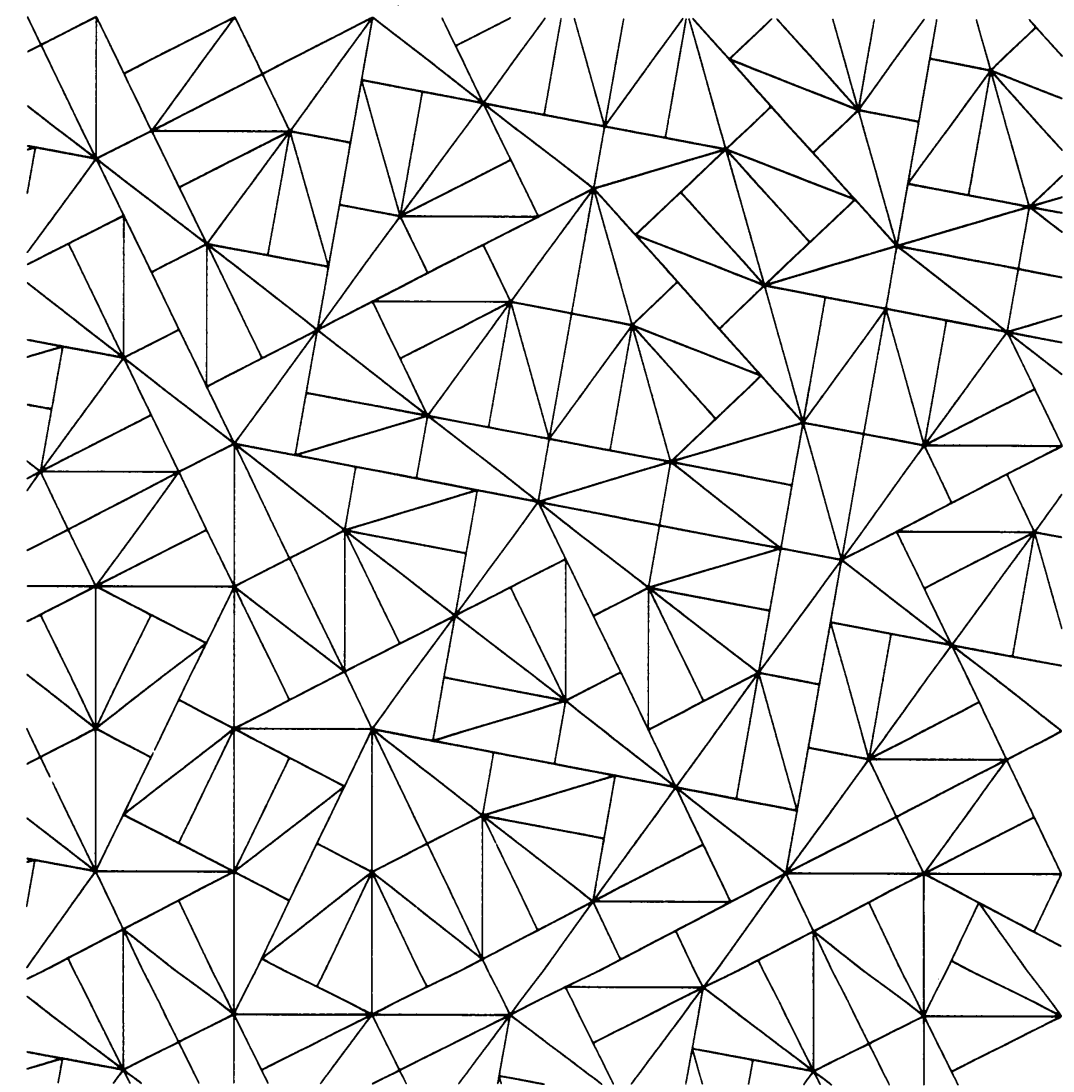

FIGURE 3

The tilings $X^{D}$ associated with the substitution function $D$ are then defined as those tilings $x$ such that each finite subcollection of tiles in $x$ is congruent to a subcollection of a set of tiles of the form $D^{r}(P)$ for some letter $P \in A$ and integer $r \geq 1$. It is important, and not hard to prove, that as a map on $X^{D}, D$ is one-to-one and onto. A portion of a tiling from $X^{D}$ appears in Figure 3. (It is instructive to compare the above geometrical constructions with the traditional subject of symbolic substitution dynamics [8].) Now this substitution dynamical system $X^{D}$ has been used $[12,13]$ to construct a tiling dynamical system $X_{F}$ which is isomorphic to $X^{D}$ as a dynamical system and inherits the symmetry $D$. This has been one of the main techniques in constructing aperiodic tilings. The best known example is the Penrose tilings, for which, however, the definition of the map $D$ is slightly more complicated [6]. 


\section{ConClusion}

The subject of aperiodic tilings originated in the study of global structures, determined by translation invariant local rules, which have no translation symmetry $[10,6]$. There have been two main methods underlying the examples: a substitution-type method, as in the Berger, Penrose and pinwheel tilings noted above, and variants of the projection method of de Bruijn [3]. We view Theorem 2 as an indication that in higher dimensions the subject, which arose because of a difference between dimensions 1 and 2, also exhibits new possibilities in dimensions higher than 2 . We further conclude that it is natural to forbid as symmetries of the tilings not only translations but all congruences other than rotations. (This is not the only way in which the two methods differ. Also, the substitution method can produce full rotational symmetry [12,13], which the projection method seems incapable of producing.) Finally, by isolating the mechanism the proof suggests that by use of maps $D$ other than similarities one might hope to generalize the subject in other ways.

\section{ACKNOWLEDGMENTS}

It is a pleasure to acknowledge useful discussions with Marjorie Senechal and Sterling Berberian.

\section{REFERENCES}

1. D. Berend and C. Radin, Are there chaotic tilings?, Comm. Math. Phys. 152 (1993), 215-219.

2. R. Berger, The undecidability of the domino problem, Mem. Amer. Math. Soc., vol. 66, Amer. Math. Soc., Providence, RI, 1966.

3. N. G. de Bruijn, Algebraic theory of Penrose's non-periodic tilings of the plane, Kon. Nederl. Akad. Wetensch. Proc. Ser. A 84 (1981), 39-66.

4. L. Danzer, A single prototile, which tiles space, but neither periodically nor quasiperiodically, University of Dortmund, 1993, preprint.

5. M. Gardner, Extraordinary nonperiodic tiling that enriches the theory of tiles, Sci. Amer. (1977), 116-119.

6. B. Grünbaum and G. C. Shephard, Tilings and patterns, Freeman, New York, 1986.

7. D. Myers, Nonrecursive tilings of the plane, II, J. Symbolic Logic 39 (1974), 286-294.

8. M. Queffélec, Substitution dynamical systems - spectral analysis, Lecture Notes in Math., vol. 1294, Springer-Verlag, Berlin and New York, 1987.

9. C. Radin, Disordered ground states of classical lattice models, Rev. Math. Phys. 3 (1991), 125-135.

10. __ Global order from local sources, Bull. Amer. Math. Soc. (N.S.) 25 (1991), 335-364.

11. _ The pinwheel tilings of the plane, Ann. of Math. (2) 139 (1994), 661-702.

12. __ Space tilings and substitutions, Geom. Dedicata (to appear).

13. __ Symmetry of tilings of the plane, Bull. Amer. Math. Soc. (N.S.) 29 (1993), 213-217.

14. C. Radin and M. Wolff, Space tilings and local isomorphism, Geom. Dedicata 42 (1992), 355-360.

15. P. Schmitt, An aperiodic prototile in space, University of Vienna, 1988, preprint.

16. H. Wang, Proving theorems by pattern recognition II, Bell Systs. Tech. J. 40 (1961), 1-41.

17. _ Notes on a class of tiling problems, Fund. Math. 82 (1975), 295-305.

Mathematics Department, The University of Texas at Austin, Austin, Texas 78712

E-mail address: radin@math.utexas.edu 